\title{
Deuterium observation in our Galaxy - View B
}

\author{
Alfred Vidal-Madjar \\ Institut d'Astrophysique de Paris, 98bis Boulevard Arago, \\ F-75014 Paris, FRANCE
}

\begin{abstract}
.
Galactic D/H evaluations from observations completed in the far UV with first the Copernicus satellite then followed by IUE and the GHRS on the HST studies already suggest that $\mathrm{D} / \mathrm{H}$ variations may be present in the interstellar medium. More recent IMAPS (the Interstellar Medium Absorption Profile Spectrograph) observations confirm that conclusion while new STIS (which replaced the GHRS on board HST) studies seem to indicate the contrary. The situation is discussed here.

Hopefully FUSE (the Far Ultraviolet Spectroscopic Explorer, launched the $24^{\text {th }}$ of June 1999) will give access to the $\mathrm{D} / \mathrm{H}$ ratio in many different galactic sites. This will help us reach a better global view of the evolution of that key element, and thus better constrain any evaluation of its primordial abundance.
\end{abstract}

\section{Introduction}

Deuterium is understood to be only produced in significant amount during primordial Big Bang nucleosynthesis (BBN) and thoroughly destroyed in stellar interiors. Deuterium is thus a key element in cosmology and in galactic chemical evolution (see e.g. Audouze \& Tinsley 1976; Gautier \& Owen 1983; VidalMadjar \& Gry 1984; Boesgaard \& Steigman 1985; Olive et al. 1990; Pagel 1992; Vangioni-Flam \& Cassé 1994; Prantzos 1996; Scully et al. 1997). Indeed, its primordial abundance is the best tracer of the baryonic density parameter of the Universe $\Omega_{B}$, and the decrease of its abundance along the galactic evolution should trace the amount of star formation (among others).

The first, although indirect, measurement of the deuterium abundance of astrophysical significance was carried out through ${ }^{3} \mathrm{He}$ evaluation in the solar wind, leading to $\mathrm{D} / \mathrm{H} \simeq 2.5 \pm 1.0 \times 10^{-5}$ (Geiss \& Reeves 1972), a value representative of 4.5 Gyrs ago. The first measurements in the interstellar medium (ISM) of the $\mathrm{D} / \mathrm{H}$ ratio, representative of the present epoch, were reported shortly thereafter (Rogerson \& York 1973). Their value of $\mathrm{D} / \mathrm{H} \simeq 1.4 \pm 0.2 \times 10^{-5}$ has, as a representative value, since then nearly not changed. For nearly three decades, these interstellar abundances have been used to constrain BBN in a direct way.

In the following, we discuss the different measurements of the deuterium abundance in the ISM within our galaxy and try to show that the situation is not that simple. 


\section{ISM observations (various approaches)}

There are several methods to measure the interstellar abundance of deuterium (see Vidal-Madjar 1991; Ferlet 1992). One of them is to observe deuterated molecules such as HD, DCN, etc... and to form the ratio of the deuterated molecule column density to its non-deuterated counterpart $\left(\mathrm{H}_{2}, \mathrm{HCN}\right.$, etc....). More than twenty different deuterated species have been identified in the ISM, with abundances relative to the non-deuterated counterpart ranging from $10^{-2}$ to $10^{-6}$. This means that fractionation effects are important, and that, as a consequence, this method cannot provide a precise estimate of the true interstellar $\mathrm{D} / \mathrm{H}$ ratio.

However, if the molecular form is HD in a place where all or nearly all the deuterium atoms are trapped in that molecule, then $\mathrm{D} / \mathrm{H}$ could be deduced reasonably well from an evaluation of the $\mathrm{HD} / \mathrm{H}_{2}$ ratio. In such cases it may be possible to evaluate the $\mathrm{D} / \mathrm{H}$ ratio as recently shown in the IR through ISO observations of the HD molecule in Orion by Bertoldi et al. (1999). They evaluated: $(\mathrm{D} / \mathrm{H})_{\text {Orion }}=7.6 \pm 2.9 \times 10^{-6}$.

More recently, it was also possible to observe with FUSE the HD molecule in the direction of a reddened star where possibly most of the deuterium is also under the HD molecular form. This observation is still very preliminary (Ferlet et al. 2000) but could lead in a near future to new estimates of the $\mathrm{D} / \mathrm{H}$ ratio in denser parts of the ISM.

Another way to derive the $\mathrm{D} / \mathrm{H}$ ratio comes through radio observations of the hyperfine line of Di at $92 \mathrm{~cm}$ (Cesarsky, Moffet \& Pasachoff 1973). The detection of this line is however extremely difficult. An interresting upper limit was derived toward Cas A (Heiles et al. 1993): $\mathrm{D} / \mathrm{H} \leq 2.1 \times 10^{-6}$ which results from a large differential fractionation of atomic $\mathrm{D}$ in molecular form.

In the galactic anticenter direction one could expect a higher $\mathrm{D} / \mathrm{H}$ values due to less stellar processing and from the searches made in that direction, the most recent one from Chengalur et al. (1997) leads to a possible detection corresponding to $(\mathrm{D} / \mathrm{H})_{\text {Gal.Anticenter }}=3.9 \pm 1.0 \times 10^{-5}$.

Finally, a detection of the Balmer $\mathrm{D} \alpha$ and $\mathrm{D} \beta$ lines in the direction of Orion was recently reporteded by Hébrard et al. (2000; see also these proceedings) showing that a new technique for evaluating $\mathrm{D} / \mathrm{H}$ may become available soon. Similar observations made in the direction of planetary nebulae lead to a new strong upper limit on the deuterium abundance: $(\mathrm{D} / \mathrm{H})_{\mathrm{NGC}} 6572 \leq 1 . \times 10^{-7}$. This shows, as expected, that deuterium is burned in stars and gives a direct observational confirmation of such a fact. This is one of the possible explanations for deuterium abundance variations.

\section{ISM observations (Lyman lines)}

Another way to derive the ISM D/H ratio is to observe the atomic transitions of the Lyman series against the background continuum of cool or hot stars.

\subsection{Cool stars}

The main advantage of observing cool stars is that they can be selected in the vicinity of the Sun. This results in low Hi column densities, and "trivial" or 
at least "simpler" lines of sight. However even with low Hi column densities of the order of $10^{19} \mathrm{~cm}^{-2}$ the presence of several interstellar components with different $b$-values implies large errors on the Hi column density, in particular when very low column density "hot" HI gas is detected as the so called circumstellar "hydrogen walls" (see Linsky 2000 in these proceedings). Moreover, the chromospheric Lyman $\alpha$ emission line of the target cool star has to be modeled to set the continuum for the interstellar absorption. Such a procedure necessarily introduces systematic errors along with all the other unknown instrumental systematics. In addition, in the "cool stars" approach, the detailed structure of the line of sight could be found only through the observation of the FeII and the MgII ions, which are unfortunately present in both HI and HII regions and thus may not trace properly the HI and Di gas. In particular, species like NI and OI could not be observed. This could lead to additional uncertainties.

For these reasons, deriving the HI column density has always been the limiting factor of accurate $\mathrm{D} / \mathrm{H}$ ratios measurements. Nevertheless, this method has provided the most precise measurement of the local $\mathrm{D} / \mathrm{H}$ ratio in the direction of Capella, using HST-GHRS:

$(\mathrm{D} / \mathrm{H})_{\text {Capella }}=1.60 \pm 0.09_{-0.10}^{+0.05} \times 10^{-5}$ (Linsky et al. , 1993; 1995).

This result was further confirmed by the re-analysis of Vidal-Madjar et al. (1998). Such an agreement and is probably due to the very high quality of the GHRS data, the high signal to noise ratio, the binary star motion giving access to the stellar Ly $\alpha$ profiles, the remarquably simple line of sight with apparently only one component and negligible perturbation by "hot" HI gas.

From several several additional cool stars observed with HST (see Linsky 2000 , these proceedings) it is however only possible to conclude that the deduced $\mathrm{D} / \mathrm{H}$ evaluations are compatible with the Capella one since none of these results are precise enough to place new constraints.

\subsection{Hot stars}

Hot stars are located further away from the Sun, so that one always has to face a higher $\mathrm{HI}$ column density and a non-trivial line of sight structure. In these cases, Di could not be detected at Ly $\alpha$, and one has to observe higher order lines, e.g. $\operatorname{Ly} \gamma, \operatorname{Ly} \delta, \operatorname{Ly} \epsilon$; hence these measurements have primarily come through Copernicus observations. The stellar continuum is however smooth at the location of the interstellar absorption and, moreover, NI and OI lines are available to probe the velocity structure of the line of sight. They were shown to be good tracers of $\mathrm{HI}$ in the ISM (Ferlet 1981; York et al. 1983) although NI may present some difficulties (Vidal-Madjar et al. 1998).

The $\mathrm{D} / \mathrm{H}$ ratios evaluated in the direction of hot stars by Copernicus range from $\sim 5 \times 10^{-6}$ to $\sim 2.5 \times 10^{-5}$. A large scatter is clearly detected and represents differences of the average $\mathrm{D} / \mathrm{H}$ ratio in the nearby galactic ISM (within $1 \mathrm{kpc}$ ), that may be as large as a factor $\simeq 4$. The essential question is : do these variations really exist?

Unfortunately from the Copernicus data alone, no one has been able to answer this question. To progress, one may have either to re-analyze all these data in a consistent way, looking for possible undetected systematics, or complete new observations in the direction of a great variety of targets. In effect each type of target will generate its own type of problems and systematics. 
As an example, one should recall that time variations of the $\mathrm{D} / \mathrm{H}$ ratio have already been reported toward $\epsilon$ Per (Gry et al. 1983), which were interpreted as due to the ejection of high velocity hydrogen atoms from the star. But since this perturbation can only enhance the $\mathrm{D} / \mathrm{H}$ ratio, it is worth noting that in at least four cases the $\mathrm{D} / \mathrm{H}$ ratio was found to be really low: $0.7 \pm 0.2 \times 10^{-5}$ and $0.65 \pm 0.3 \times 10^{-5}$ toward $\delta$ and $\epsilon$ Ori (Laurent et al. 1979); $0.8 \pm 0.2 \times 10^{-5}$ toward $\lambda$ Sco (York 1983) and $0.5 \pm 0.3 \times 10^{-5}$ toward $\theta$ Car (Allen et al. 1992). In each case, authors discussed in details possible systematics but concluded that none of the identified ones could explain such values. These low values thus seems to be real.

To make more progresses, observations were made with new space instrumentations. One with Orfeus II (Bluhm et al. 1999), in the direction of a galactic halo star lead to $\mathrm{D} / \mathrm{H}=1.2_{-0.4}^{+0.5} \times 10^{-5}$, an interresting value since it concerns a new part of the ISM where no evaluation was made before. The result is not however significatively different from the local ISM evaluation.

Other observations were made with the IMAPS instrument in the direction of stars already observed with Copernicus. The combination of these observations with a new technique to evaluate the total Hi content on the line of sight through the use of many IUE observations of the Ly $\alpha$ line, lead to three new evaluations of the average $\mathrm{D} / \mathrm{H}$ ratio in the nearby ISM (see also Sonneborn et al. in these proceedings). They are, $(\mathrm{D} / \mathrm{H})_{\delta \text { Ori A }}=0.74_{-0.13}^{+0.19} \times 10^{-5}$ (Jenkins et al. 1999), $(\mathrm{D} / \mathrm{H})_{\gamma^{2} \mathrm{Vel}}=2.14_{-0.25}^{+0.30} \times 10^{-5}$ (Sonneborn et al. 2000), $(\mathrm{D} / \mathrm{H})_{\zeta \text { Pup }}=1.39_{-0.18}^{+0.20} \times 10^{-5}$ (Sonneborn et al. 2000).

The interresting point is that the $\delta$ Ori A result confirms the Copernicus evalutaion made by Laurent et al. (1979) showing that this low average $\mathrm{D} / \mathrm{H}$ value is estimated through two different studies using different instruments. This low $\mathrm{D} / \mathrm{H}$ is also compatible with the ISO, Bertoldi et al. (1999) evaluation made in the Orion region. This seems to make that case of a low $\mathrm{D} / \mathrm{H}$ value, very strong. This average evaluation is already incompatible with the "supposed uniform" value found in the local ISM.

Note that with the same IMAPS and IUE instruments and approach, a high $\mathrm{D} / \mathrm{H}$ value is evaluated in the direction of $\gamma^{2} \mathrm{Vel}$. This is discussed in detail by Sonneborn et al. (in these proceedings). This strengthens the case of $\mathrm{D} / \mathrm{H}$ variations.

The average $\mathrm{D} / \mathrm{H}$ ratio seems thus to possibly vary by at least a factor 3 in the nearby ISM.

\subsection{White dwarfs}

Observing white dwarfs has many advantages. Such targets can be chosen near to the Sun, circumventing the main disadvantage of hot stars, and they can also be chosen in the high temperature range, so as to provide a smooth stellar profile at Ly $\alpha$. At the same time, the Ni triplet at $1200 \AA$ as well as the OI line at $1302 \AA$ would be available, allowing thus an accurate sampling of the $\mathrm{HI}$ part of the line of sight. Such observations have now been conducted using HST toward three white dwarfs: G191-B2B (Lemoine et al. 1996; Vidal-Madjar et al. 1998), Hz43 (Landsman et al. 1996) and Sirius B (Hébrard et al. 1999; see also these proceedings). Although both $\mathrm{Hz} 43$ and Sirius B D/H average evaluations are compatible with the local ISM value evaluated toward Capella, in the case 
of Sirius B, this compatibility is marginal since two components are detected on that line of sight and, imposing the "local" $\mathrm{D} / \mathrm{H}$ value to the component identified with the Local Interstellar Cloud (LIC) in which the sun is embedded, leads to a very low value in the other component. This observation may confirm that $\mathrm{D} / \mathrm{H}$ possibly varies from cloud to cloud even over few parsecs.

In the case of G191-B2B, from Lemoine et al. (1996) and Vidal-Madjar et al. (1998) studies of two independent sets of GHRS data, the line of sight velocity structure comprises one HI region, identified with the LIC observed toward Capella, together with two HII regions. If the $\mathrm{D} / \mathrm{H}$ ratio for the $\mathrm{LIC}$ is common to both the G191-B2B and Capella sight-lines (these stars are separated by only $8^{\circ}$ on the sky) and equal to $(\mathrm{D} / \mathrm{H})_{\mathrm{LIC}}=1.6 \times 10^{-5}$, then the $\mathrm{D} / \mathrm{H}$ ratio for the other components appears to be significantly lower and of the order of $(\mathrm{D} / \mathrm{H})_{H_{\mathrm{II}}}=0.9 \times 10^{-5}$.

This is a very strong case for $\mathrm{D} / \mathrm{H}$ variations since even the average value found on that line of sight is (Vidal-Madjar et al. 1998): $(\mathrm{D} / \mathrm{H})_{\mathrm{G} 191-\mathrm{B} 2 \mathrm{~B}}=$ $1.12 \pm 0.08 \times 10^{-5}$.

However since this result is contested by the recent study of Sahu et al. (1999 ; see also these proceedings) which claims from new STIS observations, that " $\mathrm{D} / \mathrm{H}$ values in both components are consistent with $(\mathrm{D} / \mathrm{H})_{\mathrm{LIC}}=(1.5 \pm$ $0.1) \times 10^{-5}$ ", I will discuss below in detail their arguments and underline the possibble cause of such a disagreement.

\section{The case of G191-B2B}

This case is interresting because the line of sight was observed with similar high signal to noise ratio with two different and excellent instruments on board HST, GHRS and STIS. Both instruments present a very high spectral resolution.

Furthermore these data sets were treated independently by three different groups using different approaches leading to different spectra:

- GHRS data were analysed by Vidal-Madjar et al. (1998) (V98) with different procedures described there in, as well as by Sahu et al. (1999) (S99) and Howk \& Sembach (2000) (H00) using each their own GHRS package;

- STIS data were analysed by S99 using a complete package developed by the STIS Instrument Team and by $\mathrm{HOO}$ who used their own package to correct for the stray light in the STIS echelle, leading to two other STIS spectra kindly forwarded to me by the two groups in order to complete a precise analysis.

\subsection{The number of components and the zero level}

S99 claimed that only two components are needed on that line of sight arguing that the STIS SirI line is shifted by $4 \mathrm{~km} / \mathrm{s}$ relative to the GHRS one. Although this point is correct, other arguments justify the need for three components along this line of sight. They are : i) the separation between the "two clearly seen" components in the SiII lines is of $10.25 \mathrm{~km} / \mathrm{s}$ while $11.00 \mathrm{~km} / \mathrm{s}$ in the NI triplet, ii) the bottom of the saturated OI line is more easily represented, close to the zero level, by the presence of a third component and iii) the width of the strongest SirI component is larger than the corresponding SirI one. All these arguments concern relative widths and spectral separations and are thus strong observational evidences. 

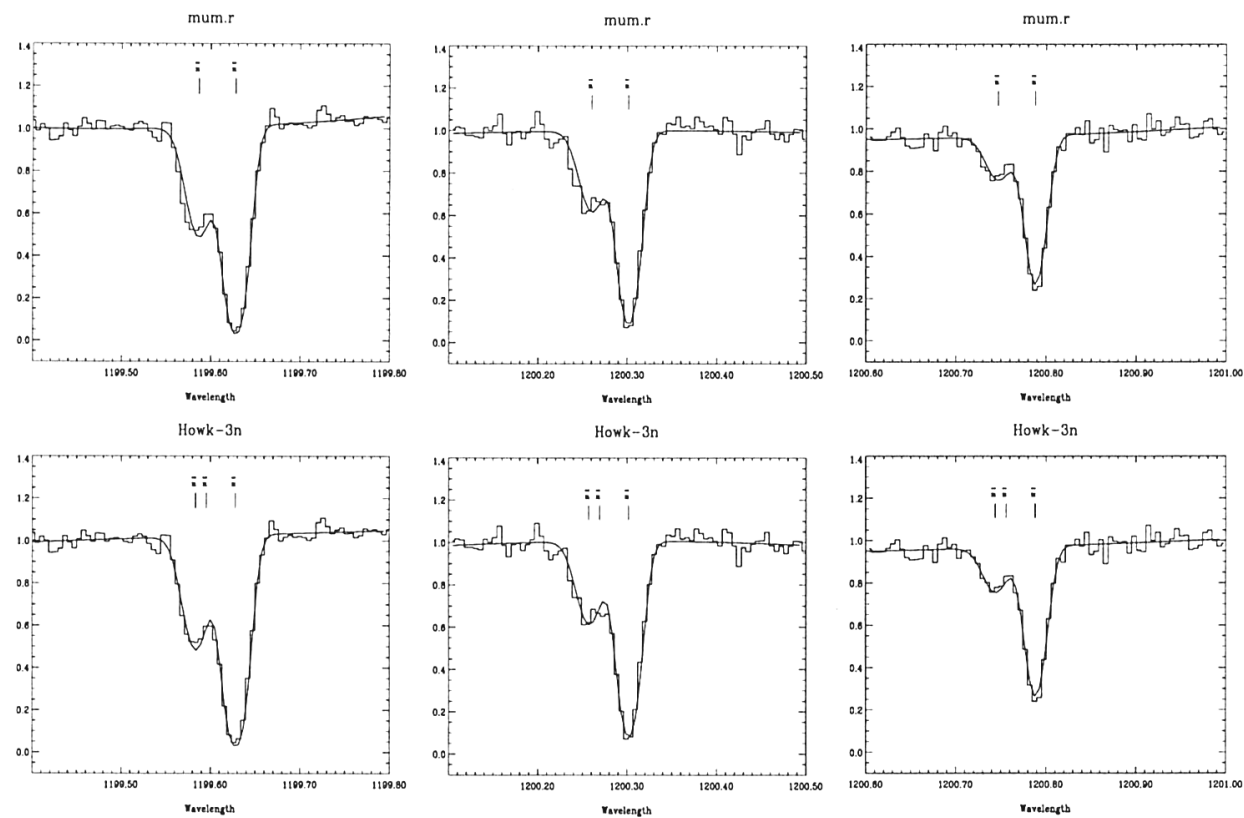

Figure 1. The STIS Ni triplet. Top, 2 components, bottom, 3 components. Fits made along with the Si lines where the "two" apparent components are closer. The 2-components fit is significantly poorer. This is not seen if one looks only at the weaker Ni line (right pannels).

Figure 1 shows that the STIS data also ask for a third component if all NI line are used.

However, as shown in Figure 2 (left), the study of S99 STIS data shows that the $\mathrm{D} / \mathrm{H}$ evaluation is insensitive to the number of components supposed, as long as more than one is assumed. It is interresting to note however that if only one component is taken into account, then $\mathrm{D} / \mathrm{H}$ is significantly different, a warning for more complex lines of sight studies. Thus having fitted the data with two or three components is not the cause of the discrepency.

It was also argued that the zero level in an echelle spectrograph is not well controlled and that is may be the cause of the disagreement. Using the set of STIS data from S99 and varying arbitrarily the zero level by few percent of the nearby continuum level, it is shown in Figure 2 (right) through the $\Delta \chi^{2}$ method (see e.g. V98) that within $\pm 2 \sigma$, i.e. conservatively when $\chi^{2}$ varies by less than 10 , this induces at most a variation on $\mathrm{D} / \mathrm{H}$ of $\pm 0.1 \times 10^{-5}$. This is also found with the other data sets and is thus not the explanation for the discrepency.

\subsection{The stellar Ly $\alpha$ continuum}

In both V98 and S99, the study of the stellar continuum was made carefuly. S99 argued that they used the best available non-LTE model (NLTE) and thus that their correction has to be the right one. V98 however tried to analyse the effect of the assumption of different types of continuum and showed that it can be adjusted in any case by the fitting procedure. So the precise knowledge of the 

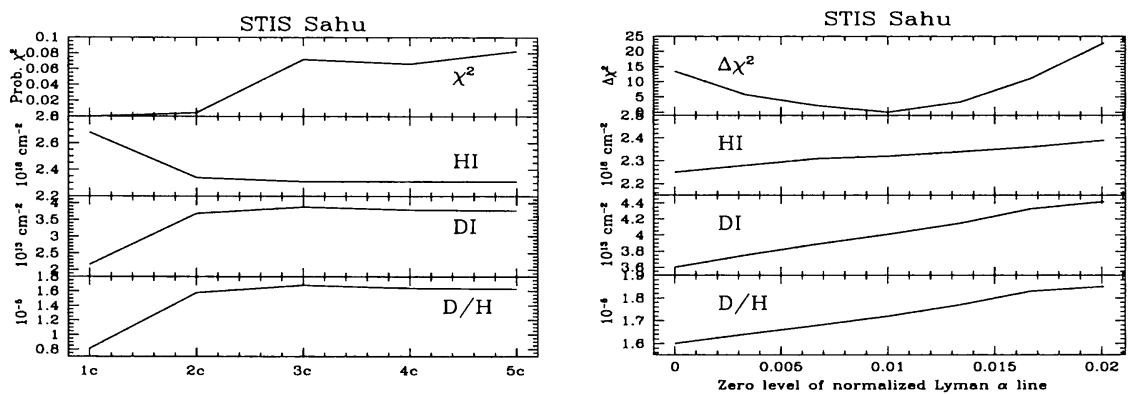

Figure 2. Left: Variation of the STIS Sahu et al. (1999) data fit solutions as a function of the number of components (c) assumed. The probability of obtaining each $\chi^{2}$ is low due to hidden systematics. However a significative jump is observed when going from 2 (2c) to 3 (3c) components. Evaluated total HI and DI column densities are shown. The average $\mathrm{D} / \mathrm{H}$ is insensitive to the number of components above 2c. This is because the $\mathrm{H}$ and $\mathrm{D}$ lines are intrinsically broad.

Right: Variation of the fitting parameters as a function of the zero level assumed at the bottom of the Lyman $\alpha \mathrm{H}$ and D lines. The "real" zero is found to be about $1 \%$ higher than the instrument corrected zero (according to Sahu et al. 1999 treatment). The $\Delta \chi^{2}$ variation relative to the best $\chi^{2}$ is shown and indicates that a poor estimate of the zero level cannot induce an error on $\mathrm{D} / \mathrm{H}$ larger than about $\pm 0.1 \times 10^{-5}$ $(\sim 2 \sigma)$.

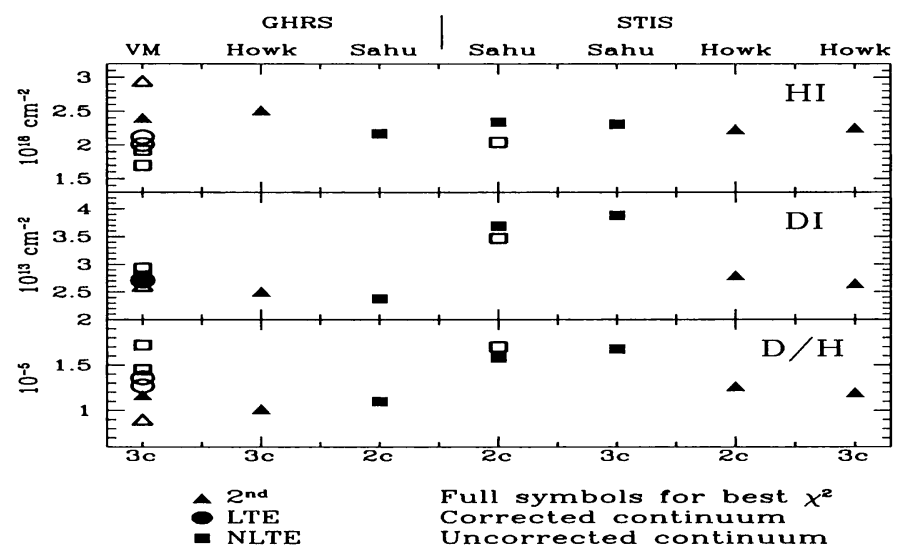

Figure 3. The HI and Di total column density on the G191-B2B line of sight as a function of the data treated : GHRS (left) from V98 (VM), H00 (Howk) and S99 (Sahu) and STIS (right) from S99 (Sahu) or H00 (Howk) (see text). The number of components assumed is indicated in the abscissa (2c or 3c). The filled symbols correspond to the best fits, in most cases obtained with corrected stellar continuum when available (see V98 and text for more details). The total HI is very stable. The disagreement comes from the Di column density deduced from the STIS data as treated by S99. All other evaluations agree together within $1.15 \pm 0.15 \times 10^{-5}$. 

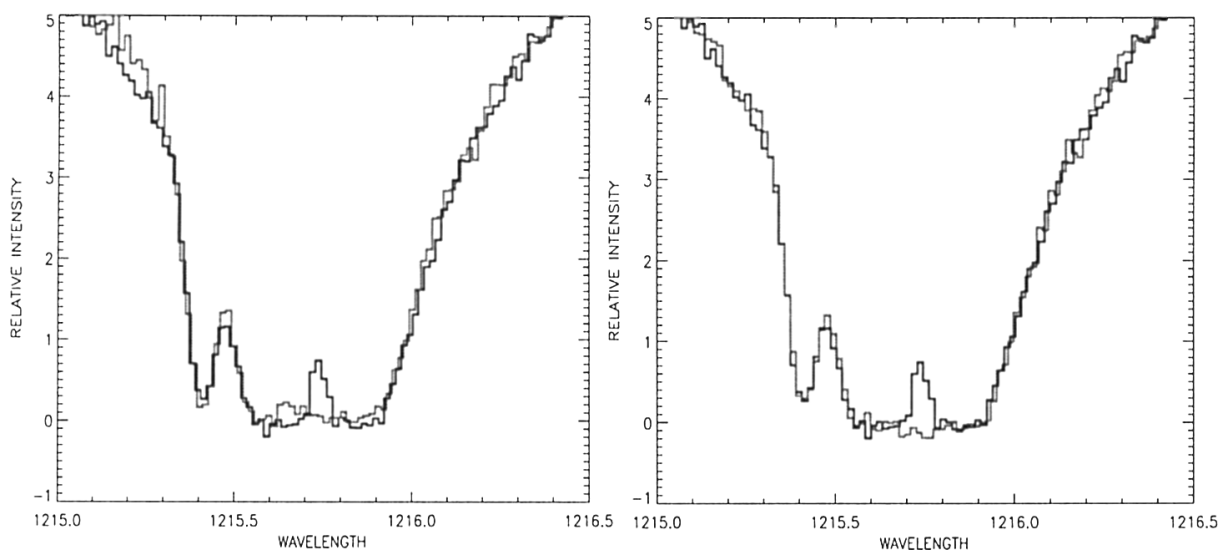

Figure 4. The comparison of the S99 (left) and the H00 (right) STIS profiles (thin histogram, binned 3 times) with the GHRS one as processed by H00 (since all GHRS studies agree together, thick histogram in both, binned 5 times) are shown (see text). In both pannels the central emmission is due to GHRS data and corresponds to the earth geocoronal Lyman $\alpha$ emission. Note the remarquable conspiracy to produce similar line shapes from two independant sets of data in the right pannel while on the left hand side, deviations could be seen in many places at $1215.2 \AA, 1215.4 \AA$ (bottom of D line), $1215.5 \AA$ (top of D line), $1215.65 \AA$ (position of earth geocorona during STIS observations), $1216.1 \AA, 1216.2 \AA$. The deviations near the D line are the cause of the discrepency between the different studies.

continuum is not too critical. To illustrate this, in Figure 3 are presented the different data sets analysed by assuming three types of stellar continua $\left(2^{\text {nd }}\right.$ order polynomial, LTE or NLTE) fixed or fitted simultaneously with the interstellar lines (see V98, for more details). The result is striking: the HI column density is not sensitive to such changes and is thus very precisely evaluated. The HI total content of the line of sight is very stable with any data set used or whatever stellar continuum assumed as long as the fitting procedure is able to adjust it (by the way one can note that the stellar continuum used by S99, is quite good since an additional adjustment of the fitting procedure does not change significantly their result).

On that same Figure 3 however, one can note that on the contrary it is the DI column density that seems to vary! It varies, not in relation with that study, but only in the case of the S 99 STIS data set. All other sets, GHRS or STIS from V98 or H00 agree very well together. The difference thus clearly comes from the detailed D line profile as in fact explained by S99.

\subsection{The $\mathrm{D}$ line profile}

In Figure 4 the GHRS and STIS line profiles are compared by binning the GHRS data five times and the STIS data three times in order to have a similar sampling. It is stricking to see how the GHRS and STIS, H00 data look similar while the GHRS and STIS, S99 data are discrepent, particularly over the deuterium line 
(see also comparison figures presented in both S99 and H00 papers). Contrary to what S99 claimed, this is not a background issue as discussed earlier, but a line profile issue since in the two different treated STIS data one (H00) looks exactly like the GHRS one while the other (S99) does not. How could a process correct the STIS data to look like the GHRS one ? Could it be a strange conspiracy or is it, more simply, that one set was corrected properly and the other not?

To strengthen that argument, note that the GHRS instrument had a very efficient procedure called FP-SPLIT able to correct for detector defects, while in these STIS data a similar procedure was not used. Also note that along the Hi Ly $\alpha$ wings, which should be smooth by definition, other fluctuations could be seen between the STIS S99 profile and the GHRS one while everywhere the GHRS and STIS H00 data nicely match together. Even in the core of the Ly $\alpha$ line, supposed to be flat, fluctuations are seen in one set and less in the other.

In conclusion, out of these four data sets, the disagreement is coming from only one set and concerns solely the $\mathrm{D}$ profile, leading to a different $\mathrm{D} / \mathrm{H}$ value. From that discussion it seems that one set of data contains probably some uncorrected instrumental fluctuations that led S99 to erroneous conclusions.

\section{Conclusion}

The different $\mathrm{D} / \mathrm{H}$ evaluations made within the galaxy lead to the fact that the $\mathrm{D} / \mathrm{H}$ ratio seems to vary from region to region for reasons still unknown as discussed in e.g. Lemoine et al. (1999).

The present observations lead to the following conclusions:

- in the local ISM $(<100 \mathrm{pc})$ average variations are of the order of $30 \%$

- in the local ISM component to component variations may reach a factor of 2

- in the nearby ISM $(<1000 \mathrm{pc})$ average variations may reach a factor of 3

- in the nearby ISM component to component variations may be even larger

This is today's situation, but now with FUSE in orbit we should have soon many more evaluations of the $\mathrm{D} / \mathrm{H}$ ratio in all the galaxy, within the halo and even in some extra galactic lines of sights.

Acknowledgments. I thank M.S. Sahu for providing me with her STIS data and J.C. Howk \& K.R. Sembach for giving me access to the same STIS data as treated with their procedure prior to publication.

\section{References}

Audouze, J., \& Tinsley, B.M. 1976, ARA\&A, 14, 43

Allen, M., Jenkins, E.B., \& Snow T.P. 1992, ApJS, 83, 261

Bertoldi, F., Timmermann, R., Rosenthal, D., Drapatz, S., \& Wright, C.M. 1999, A\&A, 346, 267

Bluhm, H., Marggraf, O., de Boer, K.S., Richter, P., \& Heber, U. 1999, A\&A, 352,287

Boesgaard, A.M., \& Steigman, G. 1985, ARA\&A, 23, 319 ApJ, 243, 161

Cesarsky, D.A., Moffet, A.T. \& Pasachoff, J.M. 1973, ApJ, 180, L1 
Chengalur, J.N., Braun, R., \& Burton, W.B. 1997, A\&A, 318, L35

Ferlet, R. 1981, A\&A, 98, L1

Ferlet, R. 1992, in IAU Symposium 150, 85

Ferlet, R., André, M., Hébrard, G., Lecavelier, A., Lemoine, M., Pineau des Forêts, G., Roueff, E., Vidal-Madjar, A., \& The FUSE Team 2000, ApJ, submitted

Gautier, D., \& Owen, T. 1983, Nature, 304, 691

Geiss, J., \& Reeves, H. 1972, A\&A, 18, 126

Gry, C., Laurent, C., \& Vidal-Madjar, A. 1983, A\&A, 124, 99

Howk, J.C. \& Sembach, K.R. 2000, AJ, in press

Hébrard, G., Mallouris, C., Ferlet, R., Koester, D., Lemoine, M., Vidal-Madjar, A., \& York, D. 1999, A\&A 350, 643

Hébrard, G., Péquignot, D., Vidal-Madjar, A., Walsh, J.R., \& Ferlet, R. 2000, accepted for publication in A\&A Letters

Heiles, C., McCullough, P., \& Glassgold, A. 1993, ApJS, 89, 271

Jenkins, E.B., Tripp, T.M., Woźniak, P.R., Sofia, U.J. \& Sonneborn, G. 1999, ApJ, 520, 182

Landsman, W., Sofia, U.J., \& Bergeron, P. 1996, in Science with the Hubble Space Telescope - II, STScI, 454

Laurent, C., Vidal-Madjar, A., \& York, D.G.: 1979, ApJ, 229, 923

Lemoine, M., et al. 1996, A\&A, 308, 601

Lemoine, M., et al. 1999, New Astronomy, 4, 231

Linsky, J., et al. 1993, ApJ, 402, 694

Linsky, J., et al. 1995, ApJ, 451, 335

Olive, K., Schramm, D., Steigman, G., \& Walker, T. 1990, Phys.Rev.Lett, B236, 454

Pagel, B., et al. 1992, MNRAS, 255, 325

Prantzos, N. 1996, A\&A, 310, 106

Rogerson, J., \& York, D. 1973, ApJ, 186, L95

Sahu, M.S., Landsman, W., Bruhweiler, F.C., Gull, T.R., Bowers, C.A., Lindler, D., Feggans, K., Barstow, M.A., Hubeny, I., \& Holberg, J.B. 1999 ApJ, 523, L159

Scully, S.T., Cassé, M., Olive, K.A., \& Vangioni-Flam, E. 1997, ApJ, 476, 521

Sonneborn, G., Tripp, T.M., Ferlet, R., Jenkins, E.B., Sofia, U.J., Vidal-Madjar, A. \& Woźniak, P.R., 2000, ApJ, submitted

Vangioni-Flam, E., \& Cassé, M. 1994, ApJ, 427, 618

Vidal-Madjar, A., Laurent, C., Bruston, P., \& Audouze, J. 1978, ApJ, 223, 589

Vidal-Madjar, A., \& Gry, C.: 1984, A\&A, 138, 285

Vidal-Madjar, A.: 1991, in Adv. Space Res., 11, 97

Vidal-Madjar, A., Lemoine, M., Ferlet, R., Hébrard, G., Koester, D., Audouze,

J., Cassé, M., Vangioni-Flam, E., \& Webb, J., 1998, A\&A, 338, 694

York, D.G. 1983, ApJ, 264, 172

York, D.G., et al. 1983, ApJ, 266, L55 